\title{
Capillary electrophoresis determination of huperzine A: A new prospective anti-dementia drug
}

\author{
Eladia M. Peña-Méndez ${ }^{1,3}$, Tomáš Hottmar ${ }^{1}$, Josef Havel $^{1}$, Jiří Patočka ${ }^{2}$ \\ ${ }^{1}$ Department of Analytical Chemistry, Faculty of Science, Masaryk University, Kotlářská 2, 61137 Brno, Czech \\ Republic \\ 2 Military Medical Academy, Department of Toxicology, 50260 Hradec Králové and Department of Radiology, \\ Faculty of Health and Social Care, University of South Bohemia, České Budějovice, Czech Republic \\ ${ }^{3}$ On leave from: Department of Analytical Chemistry, Nutrition and Food Science, University of La Laguna, \\ 38071 La Laguna, Tenerife, Spain
}

\begin{abstract}
Summary
Huperzine A is an alkaloid isolated from the Chinese plant Huperzia serrata. The absorption spectra and electrophoretic behaviour of the compound were studied. The compound shows two absorption maxima $\left(\lambda_{\max } 230\right.$ and $310 \mathrm{~nm}$ ). The protonation constant was determined by spectrophotometry and capillary zone electrophoresis (CZE) and the recommended value is $\mathrm{pK}=7.70 \pm 0.07$. A CZE method for the determination of Huperzine $\mathrm{A}$ in pharmaceutical tablets and serum has been developed. The method is based on separation of the compound as a protonated species with electrophoretic mobility determined as $50.7 \times 10^{-9} \mathrm{~m}^{2} \mathrm{~V}^{-1} \mathrm{~s}^{-1}$. The optimal separation conditions were: acetate buffer $(\mathrm{pH} 4.60 ; 20 \mathrm{mM})$ as a background electrolyte, separation voltage $20 \mathrm{kV}$, temperature $25{ }^{\circ} \mathrm{C}$, hydrodynamic injection $10 \mathrm{~s}$ and detection at wavelengths 230 and $310 \mathrm{~nm}$. The analyses were run in an uncoated fused-silica capillary with total length of $47.5 \mathrm{~cm}(39.0 \mathrm{~cm}$ to the detector) and $75 \mu \mathrm{m}$ I.D. The detection limit was found to be $70 \mu \mathrm{g} / \mathrm{l}$. The method of Huperzine A determination was applied for analysis of various pharmaceutical products. The results were found to be in good agreement with the declared amount. The precision of the method is $\pm 2.30 \%$. A method for the determination of HupA in serum was also developed with detection limit $\approx 110 \mu \mathrm{g} / \mathrm{l}$, which is applicable for pharmacokinetics and therapeutic drug monitoring of this compound.
\end{abstract}

Keywords: huperzine A - Alzheimer's disease - capillary zone electrophoresis

\section{INTRODUCTION}

Dementia is difficult to define and detect in the population. Even with the difficulties of determining prevalence and incidence, it is clear that dementia places a substantial burden on our society (Kukull and Bowen 2002). Dementia in the elderly is nowadays a major health care problem, mainly due to the progressive aging of the population. Alzheimer's disease (AD) is the most frequent type of dementia in old age. The number of patients with $\mathrm{AD}$ and related dementia treated in managed care organizations is increasing, and this trend is expected to continue. Treatment is difficult and acetylcholinesterase inhibitors are the only antidementia drugs used. However, another drug for possible future use is huperzine A (Hup A). Hup A is a potent, reversible and selective inhibitor of acetylcholinesterase with rapid absorption and penetration into the brain in animal tests. It exhibits memory-enhancing activities in animal and clinical trials. However a specific and sensitive method for Hup A determination is still awaited .

\section{ALZHEIMER'S DISEASE}

The pathology of $\mathrm{AD}$ is not well understood. Researchers have sought to understand the underlying pathophysiology of AD ever since Dr. Alois Alzheimer first described the condition in 1907. Unfortunately however, until recently, they have done so with limited success. This lack of 
clarity has deterred advancements in therapeutic drug research beyond all but the purely symptomatic treatment relief currently available (Cummings and Cole 2002).

$\mathrm{AD}$ is a progressive neurodegenerative disorder associated with a dysfunction in the cholinergic system, resulting from a deficiency in the neurotransmitter acetylcholine (Lawrence and Sahakian 1998). Acetylcholine plays a fundamental role in memory and learning. Therefore the present pharmacotherapy of this illness is focused on improvement of the cholinergic function (Mazurek 2000).

\section{CONTEMPORARY PHARMACOTHERAPY OF ALZHEIMER'S DISEASE}

There are several ways of of affecting the cholinergic system but in our clinical exercise it was sufficient to use acetylcholinesterase inhibitors (Emre 2002). Several drugs such as tacrine, donepezil, galanthamine or rivastigmine produced an enhancement of the central cholinergic function (Poirier 2002). Huperzine A as a prospective anti-dementia drug, is reported to have several advantages in terms of high efficiency and low toxicity (Bai et al. 2000).

\section{BRIEF RATING OF HUPERZINE A}

Hup A is chemically 9-amino-13-ethylidene-11methyl-4-azatricyclo (7.3.1.0(3.8) trideca-3(8),6,11trien-5-one (empirical formula $\mathrm{C}_{15} \mathrm{H}_{18} \mathrm{~N}_{2} \mathrm{O}, \mathrm{M}$. W. 242.32). It is an alkaloid isolated from the Chinese plant Huperzia serra and characterized by researchers as unsaturated sesquiterpenic compound with pyridone moiety and primary amino group (Yuan and Wei 1988). Its molecular structure can be seen in Fig. 1.

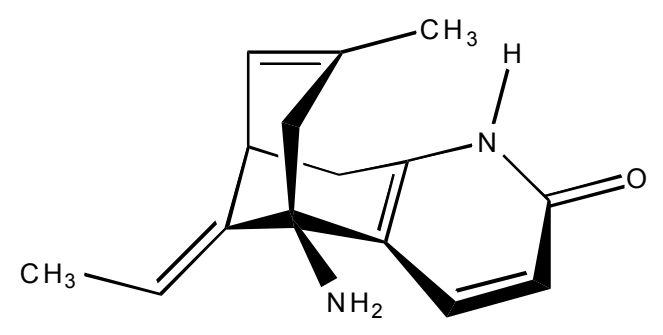

Fig. 1. Chemical structure of Huperzine A (Hup A)
The compound is optically active and in plant form its (-)-enantiomer is present and it is three times more biologically active than the synthetically prepared racemic mixture (McKinney et al. 1991). Traditional Chinese medicine has used this moss as a very popular vegetative drug for treatment of fever and inflammation (Skolnick 1997). Moreover, Hup A has been found to dramatically improve the cognitive performance of individuals suffering from various forms of memory impairment (Cheng et al. 1996) and it could be a very useful anti-dementia drug in the near future (Patočka 1999). Another interesting aspect of the pharmacological behaviour of this compound relates to its neuroprotective properties against glutamate-induced cell death:this makes this compound a potentially useful drug for reducing neuronal injury from strokes, epilepsy and other disorders (Ved et al. 1997). Grundwald et al. (1994), Ashani et al. (1996), Tonduli et al. (2001), and Lallement et al. (2002) have tested and described Hup A as a prophylactic drug against soman and other nerve gas poisoning with very good effect.

The synthesis, properties and the use of this drug are described in several papers. However, Hup A determination has not been sufficiently developed until now. The use of reverse phase HPLC to detect Hup A in plasma was described by Qian et al. (1995). However, we have not found any citations concerning the capillary electrophoretic analysis of Hup A. The aim of our work was therefore to study the Capillary Zone Electrophoresis (CZE) behaviour of Hup A as an important anticholinergic drug and if possible to develop a reliable and rapid CZE method to determine Hup A in pharmaceutical preparatives and biological fluids.

\section{MATERIAL AND METHODS}

\section{Chemicals}

The sodium acetate and phosphate were of analytical grade from Lachema (Brno, Czech Republic). The hydrochloric acid and sodium hydroxide were from Merck (Darmstadt, Germany). All chemicals were of analytical grade and were used without purification. Double distilled water obtained from a quartz distillation stand of Heraeaus Quarzschmelze (Hanau, Germany) was used to prepare all the solutions. Mesityloxide used as an electroosmotic flow marker, and benzimidazol were supplied from Fluka (Basel, Switzerland). Galanthamine was from Nivalin (Pharmachim, Bulgaria). Standard $\mathrm{pH}$ buffers were from the Institute of Serum and Vaccines (Prague, Czech Republic). A standard 
sample of (-)-Huperzine A was purchased from Panorama Research Inc. (Mountain View, CA, USA). Stock solution of Hup A: a weighed amount of the standard compound was dissolved in hydrochloric acid $(50 \mathrm{mM})$ and the solution was stored in a refrigerator. The solutions were subjected to sonification using an ultrasonic cleaner from Branson (Shelton, CT, USA). The background electrolytes (BGE) were filtered through glass crucible S4 filters from Kavalier (Sázava, Czech Republic) and degassed before use.

\section{Equipments}

A spectrophotometer ATI/UNICAM UV/Vis UV2 from Unicam (Cambridge, Great Britain) was used in all experiments. A 3DCapillary Electrophoresis Instrument from Agilent Technologies (Waldbronn, Germany) equipped with diode array detection, operating on 3D-CE CHEMStation was used for the Hup A analysis. An uncoated fused-silica capillary from Watrex (Prague, Czech Republic) with a total ength of $47.5 \mathrm{~cm}(39.0 \mathrm{~cm}$ to the detector) and $75 \mu \mathrm{m}$ I.D. was used. The background electrolyte (BGE) was acetate buffer ( $\mathrm{pH} 4.60 ; 20 \mathrm{mM})$.

Samples were injected hydrodynamically for $10 \mathrm{~s}$. A separation voltage of $20 \mathrm{kV}$ was applied (the negative polarity at the detection side) and experiments were run at a temperature of $25{ }^{\circ} \mathrm{C}$. Absorbance was monitored at 200, 230, and $310 \mathrm{~nm}$ and/or high speed scan of the spectra were used throughout the work. Mesityloxide (MSO) $0.1 \%$ $(\mathrm{v} / \mathrm{v})$ was used to determine the electroosmotic flow (EOF) under the same experimental conditions as for analysis of the samples. The capillary was washed daily for $5 \mathrm{~min}$ with double distilled water and 5 min with BGE. Between the runs the capillary was rinsed with BGE and double distilled water. The vials containing BGE were replenished after each injection. An OP-208 precision digital $\mathrm{pH}-$ meter of Radelkis (Budapest, Hungary) and $\mathrm{pH}$ sensitive combined glass electrode (Radelkis) were used for $\mathrm{pH}$ measurements. For data evaluation Statgraphics Plus V.5 package (STSC, Inc., USA) and SQUAD program (Leggett 1985) software were used.

\section{Sample Preparation}

Tablets were weighed, and added to $1 \mathrm{ml}$ of methanol and $2.5 \mathrm{ml}$ of double distilled water. This solution was sonicated for $10 \mathrm{~min}$ and then filtered through a filter with pore diameter $0.4 \mu \mathrm{m}$.

Serum was deproteinated by two different methods: a) serum $(200 \mathrm{ml})$ was mixed with acetonitrile (ratio 40:60, v/v) for $15 \mathrm{~s}$; then, the mixture was centrifuged for $1 \mathrm{~min}$ at 4000 r.p.m. and the supernatant was taken for analysis, b) serum $(200 \mathrm{ml})$ was mixed with dichloromethane (ratio 1:5, v/v) and after centrifugation an aliquot of the organic phase was taken to a separate extraction tube and Hup A was re-extracted from the dichloromethane phase into the aqueous one using $0.01 \mathrm{M} \mathrm{HCl}$ (Malovaná et al. 2001).

\section{RESULTS AND DISCUSSION}

\section{Spectrophotometry}

The absorption spectra of a standard solution of Hup A were measured in ethanol-water media (2.5\% ethanol, $\mathrm{pH}$ 6.86, I=0.1 M KCl). The spectra showed two absorption maxima at 230 and $310 \mathrm{~nm}$, respectively. Fig. 2 shows the absorption spectra as a function of $\mathrm{pH}$ in the range 2.98-9.66. It follows from Fig. 2 that there is only a small shift from 306 $\mathrm{nm}$ to $310 \mathrm{~nm}$ for the second maximum. The values of $\mathrm{pK}$ were calculated using the SQUAD program (Leggett 1985), and the result $\mathrm{pK}=7.50 \pm 0.10$ was obtained. A standard deviation of absorbance equal to \pm 0.0037 was obtained in the position of the best fit, which is in good agreement with experimental uncertainty $(\approx \pm 0.004)$. However, the standard deviation of $\mathrm{pK}$ is higher than for electrophoretic data. The reason for this is that the absorption spectra of neutral and protonated species of Hup A are very similar (Fig. 2).

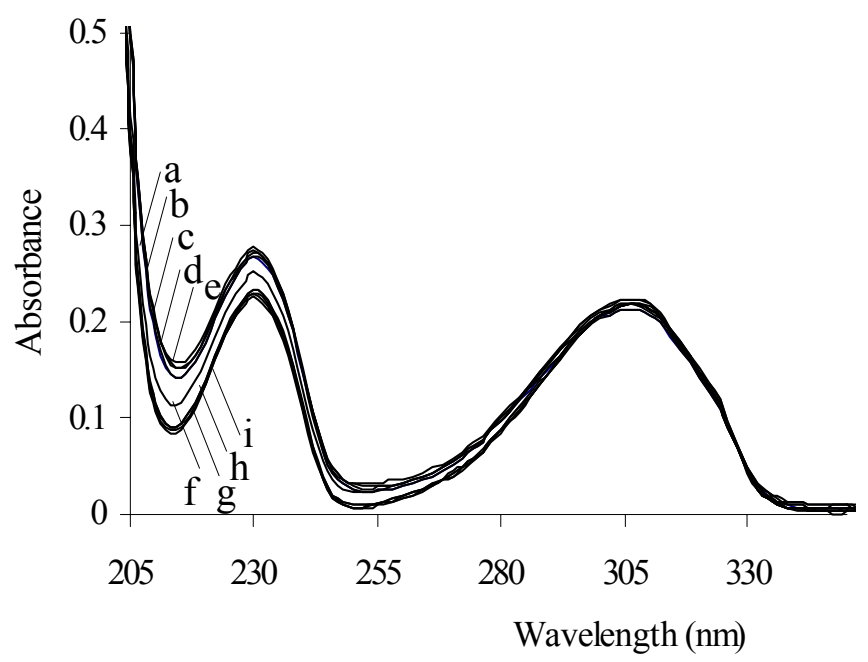

Fig. 2. The absorption spectra of Hup A $(7.3 \mu \mathrm{g} / \mathrm{ml})$ as a function of pH: a (pH 2.98), b (pH 3.63), c (pH 4.34), $\mathrm{d}$ (pH 5.34), e (pH 5.87), f (pH 6.47), g (pH 6.86), h (pH 8.12), i (pH 9.66) 


\section{Capillary Electrophoresis}

In order to optimize the electrophoretic conditions during the analysis different buffers at different concentrations, and $\mathrm{pH}$ values were tried. The dependence of effective electrophoretic mobility,

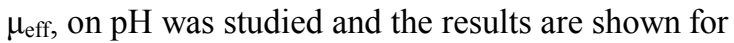
acetate buffer $(20 \mathrm{mM})$. The structure of the compound presents one $-\mathrm{NH}_{2}$ group, which can be protonated in slightly acidic media and thus cationic behaviour of Hup A during the electrophoresis may be expected. At alkaline $\mathrm{pH}$, the species is neutral. If,

$$
\text { Hup } \mathrm{A}+\mathrm{H}+\longrightarrow \mathrm{Hup}_{+} \longrightarrow(\mathrm{AH}
$$

then $\mu_{\text {eff, }}$ the effective mobility of Hup A is defined by the equation

$$
\mu_{\text {eff }}=\mu_{\mathrm{HupA}}[\mathrm{HupA}]+\mu_{\mathrm{HupAH}+}[\operatorname{Hup} \mathrm{AH}+]
$$

where $\mu_{\text {Hup }} \mathrm{A}$ and $\mu_{\text {Hup }} \mathrm{AH}^{+}$are mobilities of nonprotonated and protonated forms of Hup A, respectively. Of course, the mobility of nonprotonaterd form is equal to zero. Thus, if

$$
\text { [Hup A] }<[\operatorname{Hup} \mathrm{AH}+]
$$

then,

$$
\mu_{\mathrm{eff}}=\mu \mathrm{Hup} \mathrm{AH}+
$$

It can be seen from Fig. 3 that effective mobility,

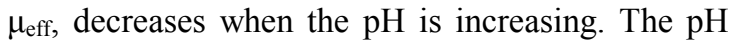
value 4.60 was found suitable for the analysis. The effective mobility as a function of $\mathrm{pH}$ confirms cationic behaviour for HupA in acid solutions. At $\mathrm{pH}$ values greater than 5.5 the effective mobility of Hup A starts to diminish and the compound starts to be neutral at $\mathrm{pH}>8$. The $\mathrm{pK}$ value of Hup $\mathrm{A}$ protonation according to equation (A) was determined using the CELET program (Havel and Jánoš 1997) and a value of $\mathrm{pK}=7.70 \pm 0.07$ was obtained. Standard deviation is lower than that obtained for spectrophotometry and because of the great similarity of Hup A and Hup $\mathrm{AH}+$ spectra, we recommend this value (7.70) as more correct one.

Evidently, for CZE determination of Hup A the acid $\mathrm{pH}$ range $(<7)$ can be used. In order to optimize the electrophoretic method, several BGE (phosphate, citrate, acetate) were tested. Different peak parameters (migration time, number of theoretical plates, symmetry, height, area, etc.) were evaluated, and finally an acetate buffer $(\mathrm{pH} 4.60 ; 20$ $\mathrm{mM}$ ) was chosen as the optimal because it shows lowest noise, greatest peak height, and short migration time. The optimal conditions with good resolution, peak shapes and short time of analysis were: acetate buffer as a background electrolyte $(\mathrm{pH}$ $4.60 ; 20 \mathrm{mM})$, separation voltage $20 \mathrm{kV}$, temperature $25{ }^{\circ} \mathrm{C}$, hydrodynamic injection for $10 \mathrm{~s}$ and detection at wavelengths 230 and $310 \mathrm{~nm}$. The reproducibility of the migration time is very good (Fig. 4). The extent of reproducibility of the migration time was obtained for 10 repeated measurements for concentration $15 \mu \mathrm{g} / \mathrm{ml}$ Hup A and the relative standard deviation value (RSD) was $1.30 \%$. Using the optimal conditions described above, the use of the method for quantitative analyses was evaluated. For the calibration curve in the concentration range $2.6-10.3 \mu \mathrm{g} / \mathrm{ml}$ of Hup A the following regression equation $\mathrm{y}=1.0357 \mathrm{x}+0.01318$ was obtained, where $\mathrm{x}$ is the concentration of Hup A. The correlation coefficient $\left(\mathrm{R}^{2}\right)$ is within the acceptable limit, $\mathrm{R}^{2}=0.9976$. The limit of detection (LOD) for Hup $\mathrm{A}$ in aqueous solution was estimated to be about $70 \mu \mathrm{g} / \mathrm{l}$ at a signal-to-noise ratio $(\mathrm{S} / \mathrm{N}=3)$. The precision assessed for $15 \mu \mathrm{g} / \mathrm{ml}$ Hup A standard error was $\pm 1.4 \%$.

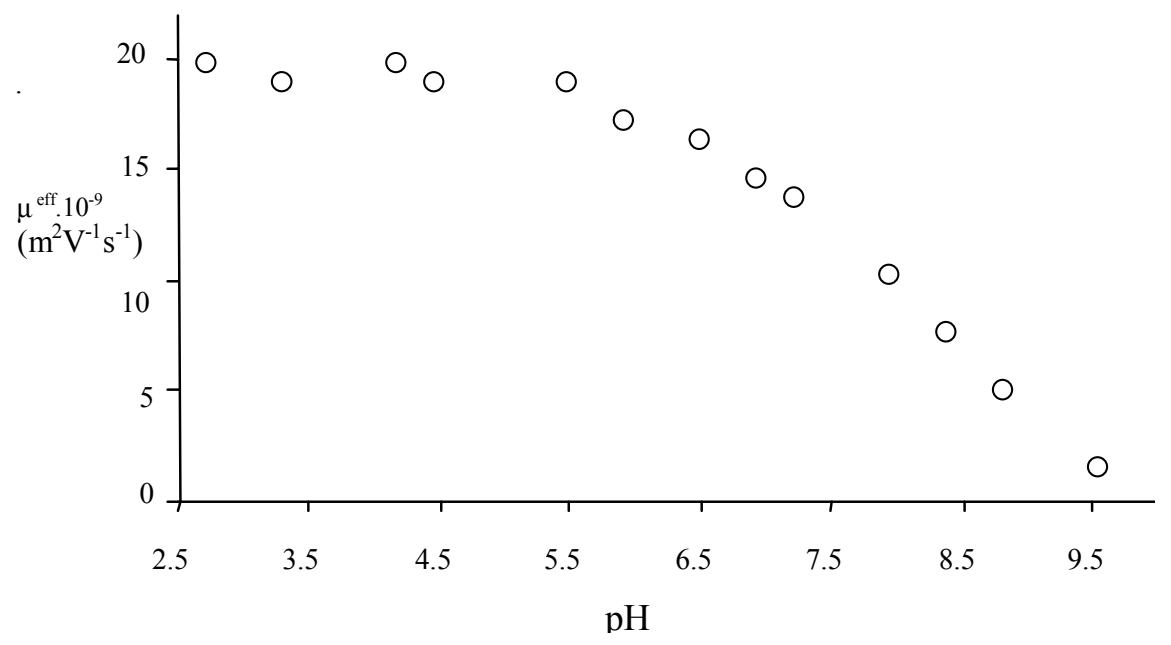

Fig. 3. Effective mobility of Hup A as a function of pH, $15 \mu \mathrm{g} / \mathrm{ml}$ Hup A. The mobility of huperzin AH+ in acetat buffer is lower, probably because of formation of ion associate with acetate anion 


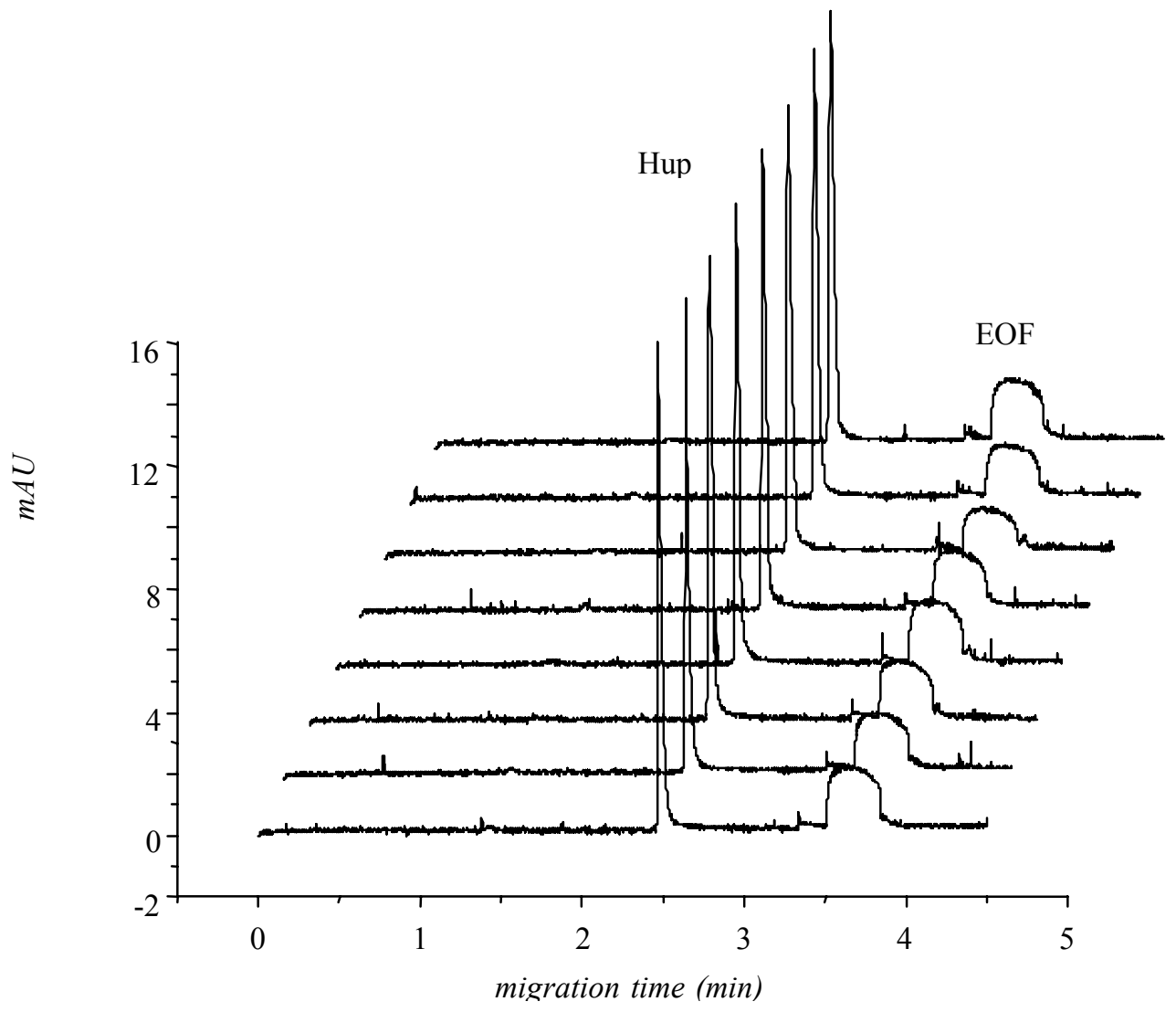

Fig. 4. Reproducibility study of the migration time of Hup A, $15 \mu \mathrm{g} / \mathrm{ml} \mathrm{Hup} \mathrm{A}$

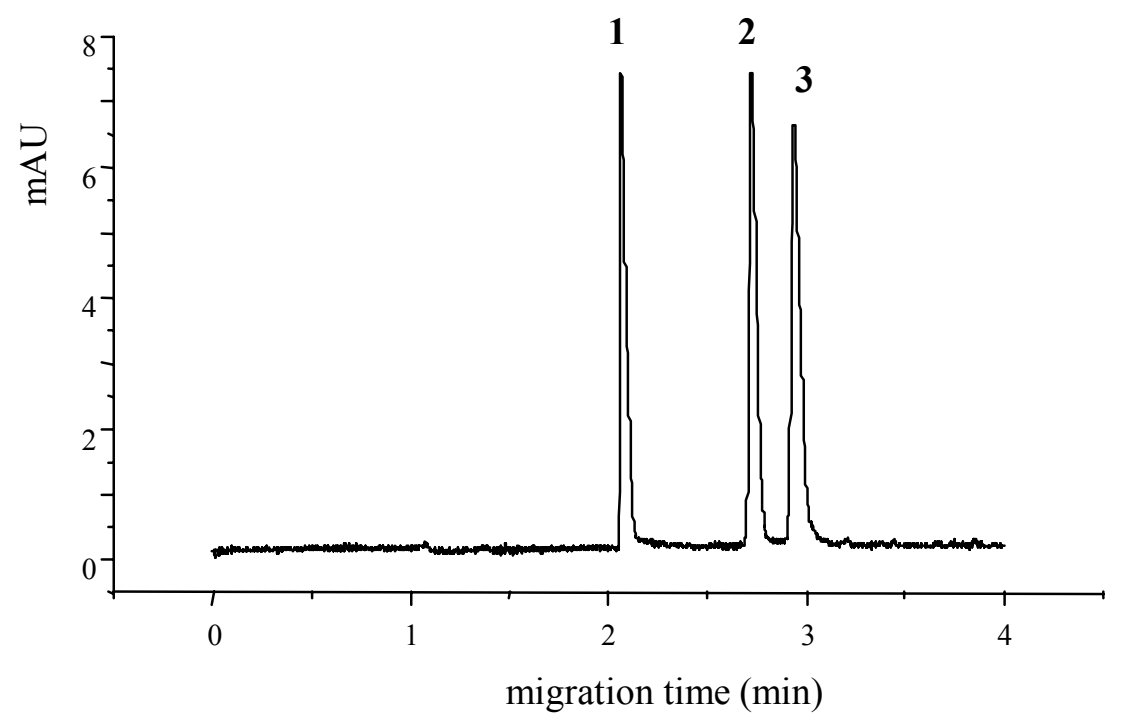

Fig. 5. The analysis of mixture (1) $16 \mu \mathrm{g} / \mathrm{ml}$ benzimidazole, (2) $16 \mu \mathrm{g} / \mathrm{ml}$ galanthamine and (3) $7 \mu \mathrm{g} / \mathrm{ml} \mathrm{Hup}$ A 
Table 1. Assay of huperzine A in commercial pharmaceutical tablets from two producers

\begin{tabular}{ccc}
\hline Tablet No. & INTLECTS $^{\text {TM }}$ Huperzine A (USA) ${ }^{*}$ & ${\text { Huperzine A Tablets }{ }^{(C h i n a)}{ }^{*}}$ \\
I & $49.9 \mu$ g per tablet & $49.2 \mu$ per tablet \\
II & 48.6 & 50.7 \\
III & 51.1 & 50.7 \\
IV & 48.4 & 48.8 \\
V & 50.5 & 48.7 \\
Average & 49.7 & 49.6 \\
S.D. & 1.2 & 1.0 \\
RSD & 2.4 & 2.0 \\
\hline
\end{tabular}

* Declared amount was $50 \mu \mathrm{g}$ of huperzine A per tablet, S.D. standard deviation, RSD relative standard deviation

The electrophoretic mobility of the protonated form of Hup A was determined as $50.7 \times 10^{-9} \mathrm{~m}^{2} \mathrm{~V}^{-1} \mathrm{~s}^{-1}$. Comparison with some other cationic drugs gives the following order: ammonium (72.2), pyridinium (51.1), Hup $\mathrm{AH}+$ (50.7), tetramethylammonium (42.6), and tetraethylammonium $\left(32.9 \times 10^{-9} \mathrm{~m}^{2} \mathrm{~V}^{-1}\right.$ $\left.\mathrm{s}^{-1}\right)$. An example of CZE analysis of the mixture of Hup A with another anti-dementia drug such as galanthamine, etc. was carried out, while benzimidazol was used as a marker. Fig. 5 shows that the migration time of protonated galanthamine is lower than the migration time of Hup $\mathrm{A} \mathrm{H}+$. Protonated huperzine migrates slower than galanthamine and its mobility is close to pyridinium.

\section{EXAMPLES OF METHOD APPLICATIONS}

\section{Analysis of tablets}

The developed method was applied for the determination of Hup A in commercial tablets. Two different types of tablets were analysed: a) The USA product - INTLECS ${ }^{\text {TM }}$ Huperzine A of Biomedisyn Corporation (Woodbridge, Connecticut, USA), b) The Chinese tablet - Huperzine A Tablets $₫$ of Joyline \& Joysun Pharmaceutical Stock Co. LTD. (China). The both products have the same declared content of $50.00 \mu \mathrm{g}$ of Hup A per tablet. The standard addition method was applied for the determination and the results of the analysis are shown in Table 1. It was found that the Hup A content determined either in the American or Chinese tablets agreed well with the declared content of $50.00 \mu \mathrm{g}$ per tablet.

\section{Determination of Hup A in Serum}

Hup A is presupposed as an anti-dementia drug in Alzheimer's disease and as an antidote against nerve gas poisoning. We have, therefore, examined also the possibility of determining Hup A in serum. A high concentration of proteins and ions in serum samples interferes generally with the analysis of small molecules. Two methods for deproteination were tested to eliminate interference from the matrix. Finally, the method developed by Malovaná et al. (2001) was chosen. After the deproteination the eletrophoretic method described above was applied for the determination of Hup A in serum. Example of an electropherogram concerning the determination of Hup A in serum is shown in Fig. 6. The detection limit was estimated to be $110 \mu \mathrm{g} / \mathrm{l}$ $(\mathrm{S} / \mathrm{N}=3)$. When Hup A was tested as an antidote against nerve gas poisoning, the research groups (Grundwald et al. 1994, Ashani et al. 1996, Tonduli et al. 2001, and Lallement et al. 2002) estimated that a dose of $500 \mu \mathrm{g} / \mathrm{kg}$ was necessary to reduce toxicity due to soman. These authors studied the effects of administration of Hup A in different animals. The applied method satisfies the required sensitivity for the determination of Hup A values in serum for the administered doses. 


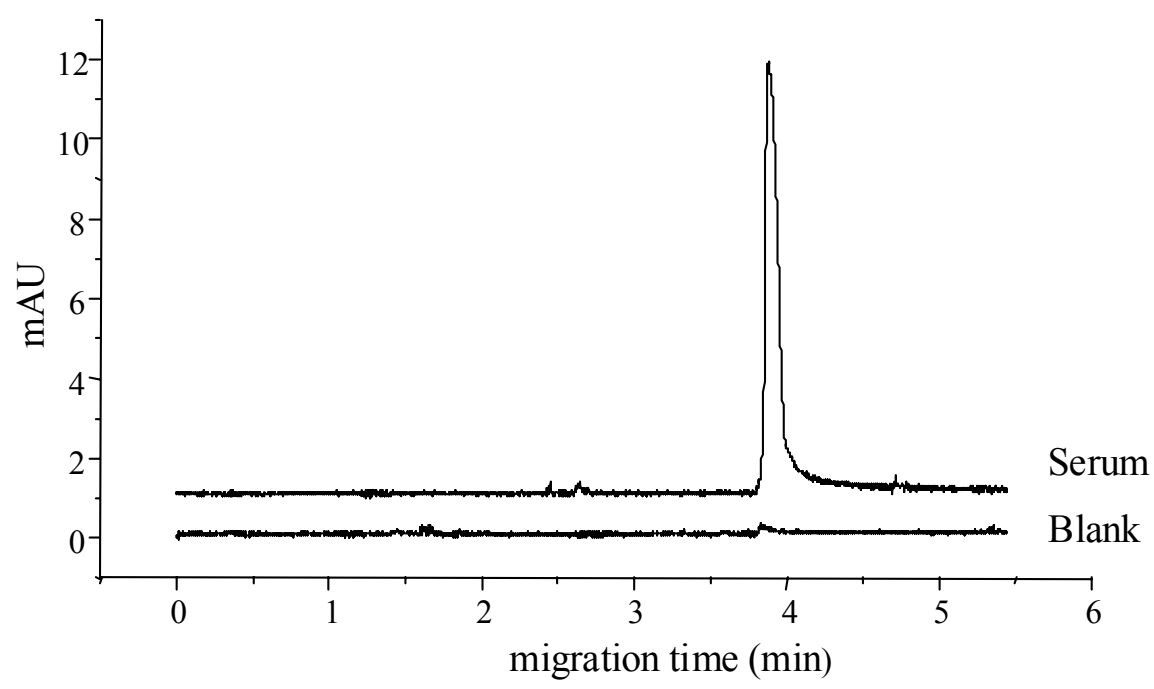

Fig. 6. An example of the electropherogram obtained during the analysis of $13 \mu \mathrm{g} / \mathrm{ml}$ Hup $A$ in the serum

\section{CONCLUSIONS}

HupA is protonated in aqueous solution with $\mathrm{pK} \approx$ 7.7 with very similar spectra of neutral and protonated forms. The protonated form of huperzine, Hup $\mathrm{AH}+$, has rather high electrophoretic mobility and this is the base of capillary electrophoretic method determination with a good detection limit $(70 \mu \mathrm{g} / \mathrm{l})$ at $230 \mathrm{~nm}$. The CZE method developed yields a good reproducibility and it was successfully applied for the determination in pharmaceutical products. It was also found that Hup A can be determined in serum with sufficient detection limit $(110 \mu \mathrm{g} / \mathrm{l})$.

\section{Acknowledgements}

Ministery of Education and Youth of the Czech Republic is acknowledged for supporting the work via project No J07/98:143100011.

Received $10^{\text {th }}$ September 2002.

Published online $28^{\text {th }}$ February 2003.

\section{REFERENCES}

Ashani Y., J. Grunwald, C. Kronman, B. Velann, A. Shafferman: Role of tyrosine 337 in the binding of huperzine $A$ to the active site of human acetylcholinesterase. Mol. Pharmacol. 45: 555-560, 1994.

Bai D.L., X..C. Tang, X.C. He: Huperzine A, a potential therapeutic agent for treatment of Alzheimer's disease. Curr. Med. Chem. 7: 355374, 2000.

Cheng D.H., H. Ren, C.C. Tang: Huperzine A, a novel promising acetylcholinesterase inhibitor. Neuroreport 8: 97-101, 1996.

Cummings J.L. and G. Cole: Alzheimer disease. J. Am.. Med. Assoc. 287: 2335-2338, 2002.

Emre M.: Switching cholinesterase inhibitors in patients with Alzheimer's disease. Int. J. Clin. Pract., Suppl. 127: 64-72, 2002.

Grunwald J., L. Raveh, B.P. Doctor, Y. Sahani: Huperzine A as a pretreatment candidate drug against nerve agent toxicity. Life Sci. 54: 991997, 1994.

Havel J. and J. Jánoš: Evaluation of capillary zone electrophoresis equilibrium data using the CELET program. J. Chromatogr A 786: 321-331, 1997.

Kukull W.A. and J. D. Bowen: Dementia epidemiology. Med. Clin. North Am. 86: 573590, 2002.

Lallement G., V. Baille, D. Baubichon, P. Carpentier, J.M. Collombet, P. Filliat, A. Foquin, E. Four, C. Masqueliez, G. Testylier, L. Tonduli, F. Dorandeu: Review of the value of huperzine as pretreatment of organophosphate poisoning. Neurotoxicology 23: 1-5, 2002.

Lawrence A.D. and B. J. Sahakian: The cognitive psychopharmacology of Alzheimer's disease: focus on cholinergic systems. Neurochem. Res. 23: 787-794, 1998. 
Leggett D.J. (ed.): Computational Methods for Determination of Formation Constants, Plenum Press, New York, 1985.

Malovaná S., D. Gajdošová, J. Benedík, J. Havel: Determination of esmolol in serum by capillary zone electrophoresis and its monitoring in course of heart surgery. J. Chromatogr. B, 760: 37-43, 2001.

Mazurek A.A.: Treatment of Alzheimer's disease. N. Engl. J. Med. 342: 821, 2000.

McKinney M., J.H. Miller, F. Yamada, W. Tuckmantel, A.P.: Kozikowski Potencies and stereoselectivities of enantiomers of huperzine A for inhibition of rat cortical acetylcholinesterase. Eur. J. Pharmacol. 203: 303-305, 1991.

Patočka J.: Může stará čínská droga pomoci pacientům s Alzheimerovou chorobou? Psychiatrie 3: 23-24, 1999.

Poirier J.: Evidence that the clinical effects of cholinesterase inhibitors are related to potency and targeting of action. Int. J. Clin. Pract., Suppl. 127: 6-19, 2002.
Qian B.C., M. Wang, Z.F. Zhou, K. Chen, R.R. Zhou, G.S. Chen: Pharmacokinetics of tablet huperzine A in six volunteers. Acta Pharmacolog. Sinica 16: 396-398, 1995.

Skolnick A.A.: Old Chinese herbal medicine used for fever yields possible new Alzheimer disease therapy. J. Amer. Med. Assoc. 277: 776, 1997.

Tonduli L,S., G. Testylier, C. Masqueliez, G. Lallement, P. Monmaur: Effects of Huperzine used as pre-treatment against soman-induced seizures. Neurotoxicology 22: 29-37, 2001.

Ved H.S., M.L. Koenig, J.R. Dave, B.P. Doktor: Huperzine A, a potential therapeutic agent for dementia, reduces neuronal cell death caused by glutamate. Neuroreport 8: 963-968, 1997.

Yuan S.Q. and T.T. Wei: Studies on the alkaloids of Huperzia serrata (Thunb.) Trev. [Article in Chinese]. Yao Xue Xue Bao 23: 516-520, 1988.

\section{Address:}

Jiří Patočka, Department of Toxicology, Military Medical Academy, Šimkova 878, 50001 Hradec Králové, Czech Republic; patocka@pmfhk.cz 